\title{
Atresias de Duodeno, Yeyuno e Ileon
}

Dres. Patricio Barraza R.*; Alberto Veloso N.*; Alberto Sálamon I.*; Patricio Vera C.*

cidos en la literatura mundial, de los que había solamente 139 (9\%) de sobrevivientes.

\section{(2) Estudio de los resultados}

Iniciamos el estudio retrospectivo de nuestros fracasos; analizamos las fichas antiguas, protocolo de operaciones, necropsias, analizamos las radiografías, inquirimos impresiones de los antigutos cirujanos tratantes, etc., y hurgando en este material encontramos muchas causas.

Habían numerosos factores adversos, que eran imposibles de modificar:

Arriarán, hasta el año 1967, inclusive.

2. Estudio de esos resultados.

3. Planificación del trabajo.

4. Análisis de los resuítados actuales.
(1) Recopilación de las Atresias de Duodeno e Intestino Delgado - 1967

Hasta el año 1967, el Hospital Arriarán mostraba una estadística muy decepcionante, respecto a resultados en el tratamiento de las atresias del tubo digestivo. Sólo 3 niños habían sobrevivido a la reparación quirúrgica, de un total de 43 operados en un período de 19 años.

Esta desalentadora experiencia -por lo demás - era de carácter universal. Evans, en 1950, publicó un total de 1.498 casos, apare-

\footnotetext{
"Servicio de Cirugía Pediátrica, Ortopedia y Traumatología, Hospital "Manuel Arriarán".
}

Rev. Chilena de Pediatría, Vol. 47, Ne 2, 1976

A) Bajo peso - Prematurez - Dismadurez.

B) Trisomías - Mongolismo.

C) Graves malformaciones concomitantes.
D) Carencia absoluta de recutsos y ambien- te apropiado para el recién nacido.
E) La cirugía actuaba sobre niños muy descompuestos pot obstrucciones dema- siado prolongadas.

(3) Planificación del trabajo:

Pero también existían factores que eran susceptibles de cambiar, y de allí partió la Planificación del trabajo, en sentido prospectivo.

a) Envío oportuno del recién nacido al Centro Quirúrgico. Se realizó divulgación de la patología quirúrgica del recién nacido entre los profesionales que tenían que ver con el neonato en la maternidad. 
Se insistió en el buen manejo y control de las alteraciones del equilibrio hidro electrolitico y ácido básico.

b) Unificación de criterios en la terapéutica quirürgica. Los niños recién nacidos con problenas de obstrucción intestinal, llegaban al Servicio de Urgencia, y allí eran intervenidos por el médico de turno, de acuerdo a sus ideas $y$ experiencias particulares. En ocasiones incluso debían actuar ortopedistas en la solución de estos cuadros.

Se decidió formar el equipo médico de Cirugía del Recién Nacido, que posteriormente se completó con enfermeras y personal adiestrado. Desde 1972 nos asociamos con el Departamento de Neonatología.

c) Diagnóstico diferencial precoz. Muchos de los casos anteriores a 1968 llegaban tarde a la cirugía, porque estando hospitalizados, se pretendía la precisión diagnóstica, que retardaba varios días la solución del problema.

Para obviar esta situación, el equipo de recién nacido acudía de urgencia ante cualquier duda de diagnóstico que se les presentara a los médicos de tumo.

El concepto erróneo de considerar la eliminación de meconio, como sinónjmo de permeabilidad del tubo gastro-intestinal se desechó.

Los estudios radiológicos con contraste de bario se suprimieron por los problemas operatorios que creaban.

Se proscribieron los enemas evacuantes y similares porque confundían los hechos clini$\cos$ y radiologicos del abdomien.

Se impuso en cambio el diagnóstico de obstrucción intestinal, dándole máxima importancia al "vómito bilioso".

Se adoptó el aire como medio de contraste para estudio radiológico.

Los enemas, tactos rectales, fueron reemplazados por la pesquisa del gas rectal, mediante un procedimiento que dificultamos sea original, pero que no hemos encontrado des. crito. Consiste en auscultar el gas que existe normalmente en el recto de los recién nacidos con intestino indemne.

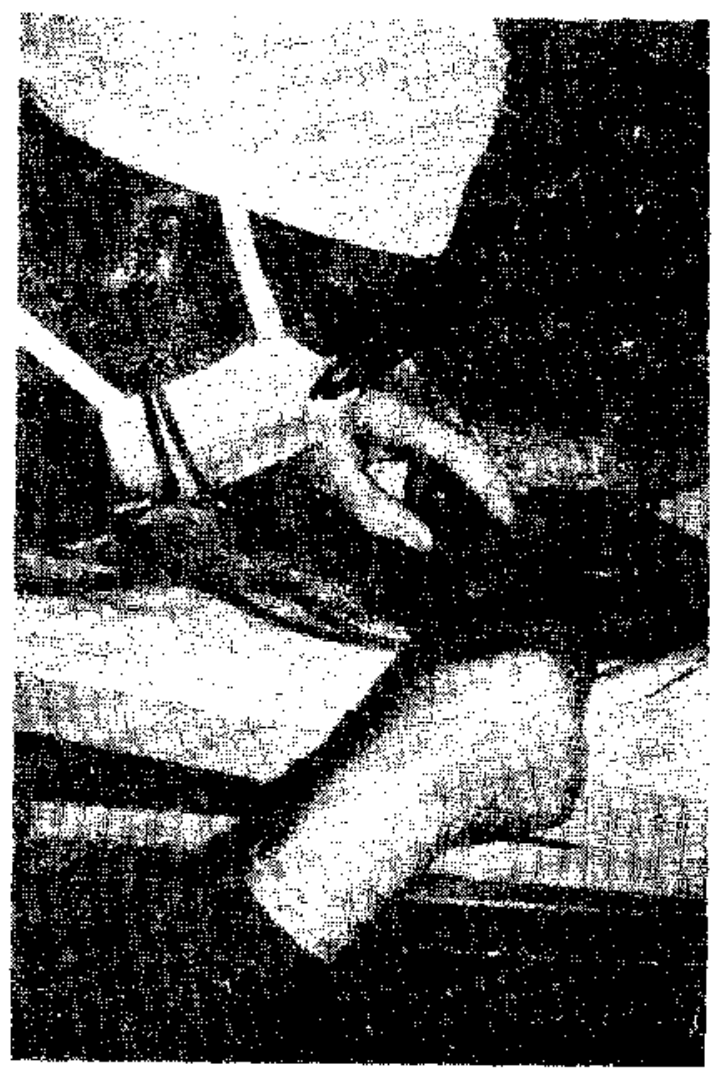

Figura $N^{\circ} 1$

Método para pesquisar el gas de la ampolla tectal por medio de una sonda Foley número 15. Util en el diagnóstico de obstrucción intestinal completa y en la yaloración de la permeabilidad de una anastomosis.

Se utiliza una sonda Nélaton $\mathrm{N}$ : 15 , con un extremo distal levemente envaselinado; el extreno proximal o bulbo se conecta herméticamente al conducto auditivo externo del examinador. La punta se introduce con suavidad en el recto y además se comprime el abdomen del nin̄o, para aumentar la presión intraabdominal.

La existencia de gas se ausculta claramente contra el tímpano del examinador; luego que se ha traspasado la barrera tónica del esfínter. Esto descarta la obstrucción intestinal completa.

En los casos de "megacolon congénito", la prueba es intensamente positiva.

Naturalmente, el examen negativo no es 
sinónimo de obstrucción intestinal, pero sí lo sugiere, al resultar reiteradamente negativo.

d) Mejoría de las condiciones materiales. Se acondicionó una sala con 2 incubadoras dotadas de oxígeno y aspiración central para el preoperatorio y postoperatorio precoz.

Termorreguladores eléctricos para la mesa de operación.

Aparatos de anestesia para el recién nacido (Blunquist).

Ventiladores mecánicos tipo Bird, con circuito " $f$ ". Tuberia y sondas adecuadas para prematuros.

Perfusores especiales de $100 \mathrm{cc}$. y fraccionadores de gotas del orden de $3 \times 1$, y $10 \times 1$ gota.

Contamos con radiografías dentro de la incubadora, to cual es de valor incalculable.

En el presente año, adquirimos bombas de infusión continua, que permiten total exactitud en el aporte líquido.

Por último, el instrumental quirúrgico es especial para este tipo de cirugía, habiendo formado cajas, con instrumental propio, y otro adaptado de vascular, oftalmología, etc.

La alimentación parenteral que en un principio consideramos la gran solución en esta cirugía, hoy sabemos que, por lo engorroso y caro, es la gran excepción. Tenemos que declarat que no disponemos de micro-métodos y esto nos obliga a prescindir al máximo de exámenes de laboratorio, y como se mostrará en algunos casos tipo; nos exige cautelar al máximo las pérdidas y egresos, obligándonos a la alimentación precoz, para evitar la acidosis de ayuno que rápidamente complica a estos pequeños pacientes operados.

\section{TECNICA QUIRURGICA}

De la revision de los casos tratados antes de 1968, concluimos:

19) El problema quirúrgico mayor lo constituía la enorme desproporción de las asas intestinales a anastomosarse. Ello habia sido solucionado de acuerdo a lo que recomenda. ban los autores clásicos, como Gross y otros:

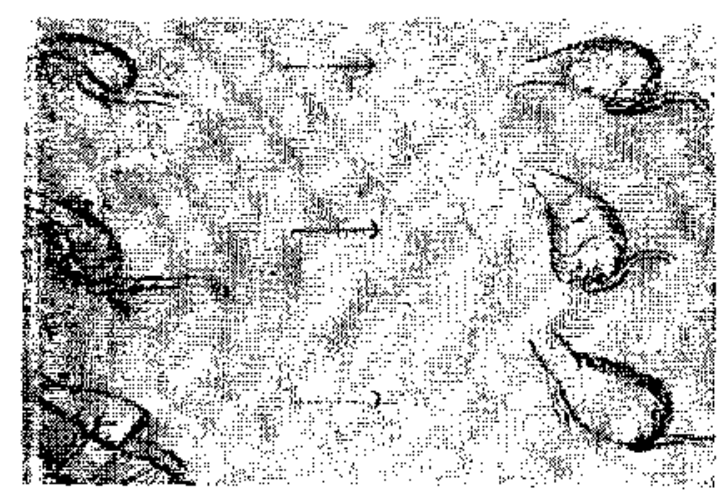

Figura No 2

Problemas técnicos de las énteroanastomosis. A la izquierda están dibujadas las soluciones quirúrgicas recomendadas en los textos clásicos de cirugía pediátrica. A la derecha, el resultado anátomo-funcional que recogimos en la investigación restrospectiva: obstrucción secundaria por asa ciega funcional.

$$
\begin{aligned}
= & \text { Anastomosis látero-laterales. } \\
= & \text { Término-laterales. } \\
= & \text { Oblicuas. } \\
= & \text { Ocasionalmente término-terminales, } \\
& \text { con cierre del excedente proximal. }
\end{aligned}
$$

En los últimos años se resecaba parcialmente el segmento dilatado, con lo cual se simplificaba en parte la intervención.

$2^{\circ}$ ) Todas las suturas practicadas hasta entonces se hicieron en 2 planos, y en oportunidades en 3 planos.

Las necropsias de los pacientes demostraron que, a pesar de que en un $1 / 3$ de los casos, la anastomosis era permeable, no había habido pasaje de contenido intestinal por constituirse en todos un síndrome de "asa ciega". La impulsión de la onda peristáltica contra la zona dilatada provocaba mayor distensión de ésta, la cual por su peso caía encima del asa eferente, aplastándola y obstruyéndola secundariamente. 
En los otros 2/3 de los casos había tal densidad de planos y de puntos en la línea de sutura, que la anastomosis estaba cerrada, y no había paso hacia distal.

Estas evidencias anatómicas y fisiológicas nos indujeron a cambiar la técnica, y desde 1968 se adoptó la siguiente:

a) Resección amplia del asa proximal distendida.

b) Anastomosis término-terminal siempre, salvo en las atresias de duodeno.

c) Sutura en un solo plano, exclusivamente, con puntos sero musculares, separados, con seda atraumática de 5 ó 6 ceros (16 a 24 puntos).

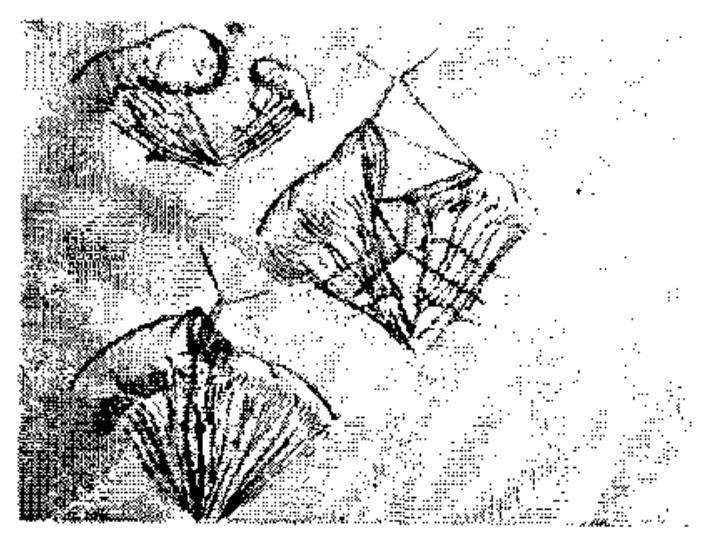

Figura Ni 3

Resumen gráfico de la técnica quirúrgica actual empleada desde el año 1968. Resección amplia del asa proximal dilatada y anastomosis término-tetminal en un plano (seromuscular).

Esta nueva técnica nos permite la alinnentación precoz a las $24 \delta 36$ horas, de acuerdo a los controles clínicos, radiológicos y fundamentalmente a la pesquisa del aire rectal, rápidamente se corrigen los trastornos hidroelectrolíticos y la recuperación del niño es general.

Toda obstrucción intestinal en el recién nacido la abordamos a través de una laparotomía transversal supraumbilical derecho, con electrobisturí. Se cateteriza la vena umbilical.

Ubicada la zona atrésica, se exterioriza el asa proximal dilatada, la cual se descomprime por aspiración. La exploración del asa distal desfuncionalizada la realizamos exclusivamente por inyección de suero glucosado tibio, sin aire, ya que podría alterar el valor de la pesquisa de gas rectal en el postoperatorio. No realizamos otro típo de distensión del asa.

En los casos de atresia del cluodeno, usamos la operación de Shunt isoperistáltico, con sutura monoplano transmesocólica o antecólica.

Hemos comprobado superioridad de la duodeno-anastomosis sobre la gastroenteroanastomosis.

Esta intervención se completa con gastrostomía por contrabertura, de dentro afuera, y la sonda se usa para intubación transanastomótica permanente por 5 a 10 días.

Esto permite instilar al yeyuno el residuo gástrico obtenido por aspiración del estómago.

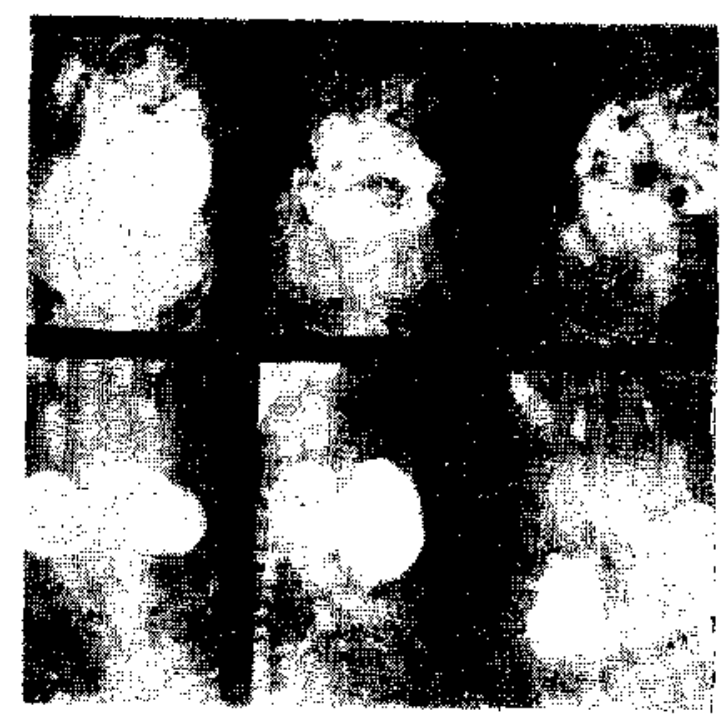

Figura N: 4

Airesia del duodeno en su segunda porción. Estudios radiológicos contrastados usando Dionosil $\mathrm{R}$. En las tres placas superiores se aprecia la oclusión completa del duodeno. En las thes radiografías inferiores se ve la sonda de silastic usada como intubación transanastomótica desde la gastrostomía, para inoculación del contenido gástrico al yeyuno.

La variante clínica de más difícil tratamiento en nuestra serie actual, la representa la atresia del asa fija, porque en ella es imposible la resección del segmento dilatado. Par- 
cialmente puede obviarse por medio de la intubación transanastomóticas, pero a tste nive1 es muy difícil el paso de la sonda desde el estómago.

El problema reside en la lenta involución del duodeno y yeyuno dilatados, y es esto de tal magnitud en contra de la recuperación de los enfermos, que nos hace plantearnos el beneficio que representaría agregar un procedimiento de vaciamiento gástrico (gastroenteroanastomosis).

No tememos a una segunda sutura, pero sí a la oportunidad en que debería realizarse la derivación por el hecho de que la mitad de los niños no la hubieran necesitado.

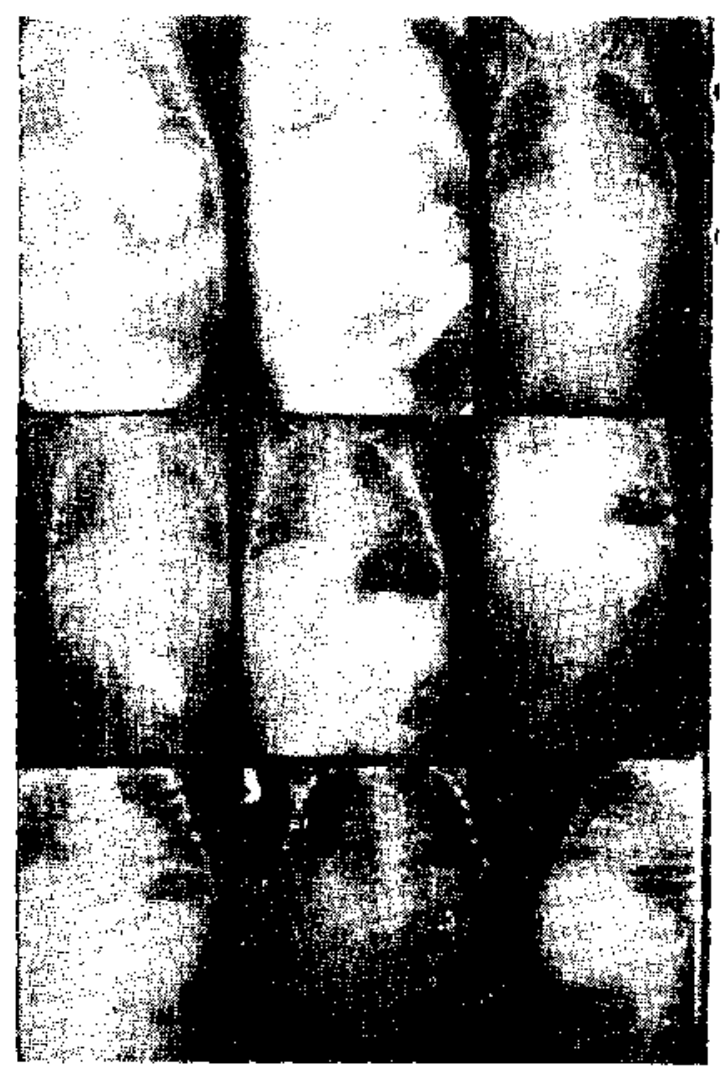

Figura No 5

Atresia del asa fija. Ingtesa con tránsito hecho con bario.

1 y 2: Tránsito al ingreso, 3: Radiografía de abdomen simple a las cuarenta y ocho horas de postoperado, muestra dos tapones de bario a nivel de la boca anastomotica. 4 y 5: Al décimo día en posición horizontal y en vertical el bario se ha fundido en uno, y clínicamente persiste el cuadro suboclusivo. 6: Reducción del tamafio del tapón de bario en el día quince. 7, 8 y 9; Resultado final al mes de haber sido intervenido.

Las atresias del yeyuno medio, distal o íleon, han sido, técnicamente, más fáciles de realizar; pero, funcionalmente, son más difíciles, retardando así la administración de alimentos.

\section{DETALLES DE TECNICA}

La sección del asa distal en ángulo de $45^{\circ}$ debe ser muy exacta, $y$ de preferencia en menor grado, porque tiene el riesgo de transformar la anastomosis término-terminal en una tếrmino-lateral.

Hemos debido reintervenir dos casos por Ealla funcional, 6 y 12 meses después de corregida la atresia.

Las atresias del tipo diafragma, en la actualidad las tratamos resecando el intestino pre y post diafragma, ya que la recuperación del peristaltismo ha resultado mejor con este procedimiento.

Apoyamos totalmente las ideas de Phelan y Nixon, que imputan todas las fallas de anastomosis al asa proximal dilatada y no a la eferente desfuncionalizada.

\section{CASUISTICA Y RESULTADO (1968 - 1975)}

Por tratarse el presente estudio del resultado de una técnica quirúrgica, hemos considerado "sobrevida" al primer mes desde la fecha de la operación.

Están incluidas en la serie de atresias, 2 estenosis muy intensas de duodeno, operadas a $\operatorname{los} 10$ y 12 días.

Hay 5 casos de atresias múltiples de intestino delgado, 3 de los cuales no tuvieron mayores problemas. 


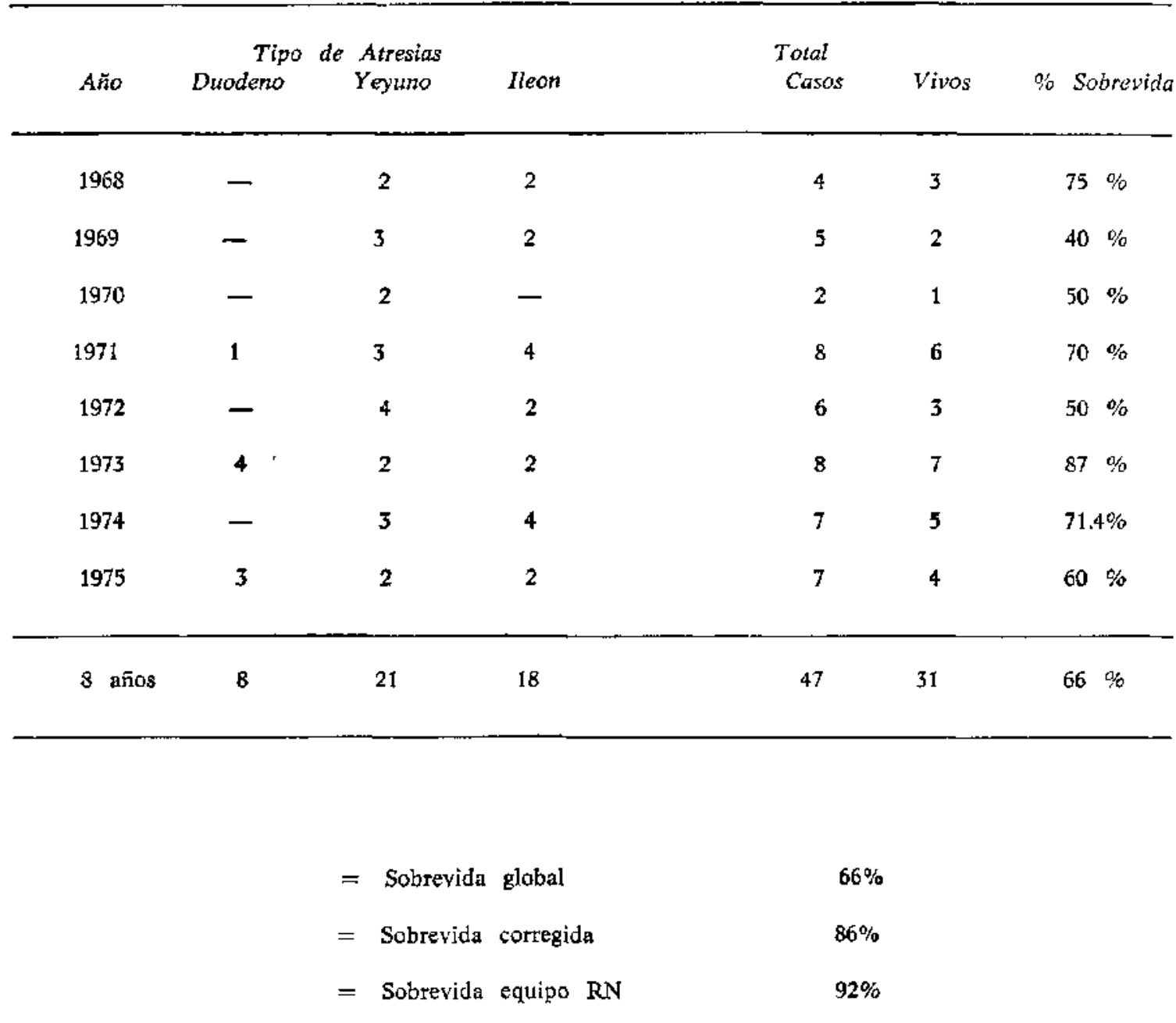

La sobrevida global del $66 \%$ debe ajustarse restando los casos que además tenían graves factores negativos asociados. En nuestra serie son 9 casos y son los siguientes:

Malformaciones congénitas incompatibles con la vida, atresia cordonal generalizada del intestino $=(1)$; atresia múltiple $=(2)$; extensas peritonitis meconiales $=(2)$; Lúes $=(1)$; Sepsis preoperatoria $\mathrm{y}$ más de 7 días de vi$\mathrm{da}=(2), \mathrm{y}$ peritonitis fecal preoperatoria $\mathrm{y}$ onfalocele roto, con necrosis masiva del intestino $=(1)$.
Total: 9 casos $=86 \%$ de sobrevida corregida. Entre las causas evitables que provocaron muertes, anotamos:

1 Caso por embolía masiva al desprenderse trombos del cateter de denudación umbilical.

2 Casos por aspiración de vómitos, en el postoperatorio inmediato.

1 Caso por caquexia secundaria a estenosis de la anastomosis al exagerar los puntos de refuerzo. 
1 Caso por coagulación intravascular y Sepsis.

1 Caso por septicemia postoperatoria.

1 Caso por oclusión extrínseca del asa distal (hernia en el anillo del mesocolon, en una gastroenteroanastomosis, en que no se ancló dicho meso).

Total: 7 casos.

En estos pacientes existía permeabilidad y funcionamiento de la anastomosis.

Al considerar los casos tratados exclusivamente por el equipo quirúrgico del recién nacido, la sobrevida corregida asciende al $92 \%$.

\section{RESUMEN Y CONCLUSIONES}

Una mortalidad superior al $90 \%$ se registraba hasta 1968 en el tratamiento quirúrgico en las atresias de duodeno y de intestino delgado en el Hospital Arriarán de Santiago de Chile. Ese año se inicia un trabajo prospectivo a 10 años plazo. En el período 1968-1975 se obtiene una sobrevida corregida de más de un $90 \%$.

Los excelentes resultados obtenidos se explican, a juicio de los autores, en base a:

1. Formación de un equibo médico y paramédico para la atencion del $\mathrm{RN}$.

2. Disponibilidad de un ambiente $y$ recursos materiales apropiados para el manejo del neonato.

3. Enfoque clínico dinámico, imponiendo el diagnóstico de obstrucción intestinal. sin agotar el estudio diagnóstico preoveratorio.

4. Uso de una técnica quirútgica apsopiada.

\section{SUMMARY AND CONCLUSIONS}

According to the records (in the Hospital M. Arriaran) mortality was over $90 \%$ in the group of patients treated for atresias of duo- denum and small intestíne, before 1968. Between 1968 and 1975, the figure has been reversed: over $90 \%$ of good results.

Surgical details have been carefully reviewed over a ten-year-period, on 47 patients.

This dramatic improvement of the general outlook can be explained by the following facts:

1) A competent group of doctors and nurses to take care of the newborns was organized.

2) Adequate hospital facilities were provided.

3) "Dynamic clinical approach", that is to say, promptness in making a surgical decision.

4) Modern surgical techniques.

\section{REFERENCIAS}

1. Renson (C. D.): Pediatric Surgery, Year Book, Chicago, 1962, volumen 2.

2. Clatworthy, H. W. and cols.: Surgery, 32: 341 1952.

3. Cubillos, $y$. y cols.: Suturas gastrointestinales en uno y dos planos. XXXVIII Congreso Chileno de Cirugía. XII-64. Tomo del Congreso, 378.

4. Daudet (M.). Chappris (I. P.) et Marion (J.): Ann. Chir. Inf., 1972, 13, 5, 291.

5. Evans, C. H.: Collective review: Atresias of gastrointestinal tract, Internat. Abstr. Surg. 92: 1, 1951, in Surg., Gynec., and Obst. Jan. 1951.

6. Gross (R.): The Surgery of Infancy and child. hood, Saunders, Philadelphia and London, 1953.

7. Herzag (B.): Progress in Pediatric Surgery, vol. 5. 1973, págs. 37 a 59.

8. Holder, T. M., and Gross, R. E.: Pediatric, $26,36,1960$.

9. Louw, J. H.: Ann. Roy. Coll. Surg. England, 25: $209,1959$.

10. Louw, 7. H., and Barnard, C. N.: Lancet, 2: $1065,1955$.

11. Lym $H$. B., and Espinas, E. E.: A.M.A. Arch. Surg. 79: 357, 1959.

12. Nixon, H. H.: Arch. Dis., childhood. 30: 13, 1955.

13. Talbert, J. L.; Felman, A. H., and De Busk, F. L.: J. Pediat., 76: 783, 1970.

14. Zikovic (S.) et Rakic (D.): Ann. Chir. Inf., 1974, $15,2,149$. 